\title{
Mécanismes de la redistribution post-mortem des xénobiotiques : le point sur l'état actuel des connaissances
}

\section{Mechanisms underlying postmortem redistri- bution of drugs : about current knowledges}

\section{Anne-Laure PELISSIER-ALICOT*(1), Jean-Michel GAULIER ${ }^{(2)}$, Pierre CHAMPSAUR ${ }^{(3)}$, Pierre MARQUET ${ }^{(2)}$}

(1) Service de Médecine Légale, Faculté de Médecine - 13385 MARSEILLE Cedex 5 (2) Service de Pharmacologie et Toxicologie, Hôpital Universitaire Dupuytren - 87042 LIMOGES

(3) Laboratoire d'Anatomie, Faculté de Médecine - 13385 MARSEILLE Cedex 5

*Auteur à qui adresser la correspondance : Anne-Laure PELISSIER-ALICOT, Service de Médecine Légale, Faculté de Médecine, 27, boulevard Jean Moulin - 13385 MARSEILLE Cedex 5 Tél : 0491324516 - Fax : 0491324512 - e-mail : apelissier@netcourrier.com

(Reçu le 5 novembre 2000 ; accepté le 10 janvier 2001)

\section{RÉSUMÉ}

Les concentrations des molécules obtenues à partir d'échantillons post-mortem ne sont pas forcément le reflet des concentrations au moment du décès. Ces concentrations post-mortem peuvent varier soit en fonction des différents sites de prélèvement, soit en fonction du délai entre le décès et la réalisation des prélèvements. Ces phénomènes ont été regroupés sous le terme de redistribution post-mortem. Les mécanismes présidant à ces phénomènes de redistribution sont complexes et multifactoriels. Ils dépendent en premier lieu de l'évolution des phénomènes cadavériques. En période post-mortem précoce, certains organes, qualifiés "d'organes réservoirs" peuvent relarguer les xénobiotiques concentrés avant le décès. Il s'agit essentiellement du tractus gastro-intestinal, du foie, des poumons ou du myocarde. La lyse cellulaire et plus tardivement la putréfaction favorisent également les phénomènes de redistribution. Le profil physico-chimique et pharmacocinétique des xénobiotiques semble être également un facteur déterminant, mais ces aspects sont moins bien connus. La seule certitude actuelle

\section{SUMMARY}

Postmortem concentrations of drugs do not necessarily reflect their concentrations at the time of death. During the postmortem period drug levels may vary according to the sampling site and the interval between death and the time of sample. These site-and time-dependent variations are called "postmortem redistribution". The underlying mechanisms are complicated depending on postmortem changes as well as on pharmacokinetic parameters. Passive drug release from drug reservoirs (e.g. gastro-intestinal tract, liver, lungs, myocardium) may occur immediately after death. Later, autolysis and putrefactive processes participate in the redistribution. Concerning pharmacokinetics, there is some evidence that basic lipophilic drugs with a large volume of distribution show important postmortem redistribution. Nevertheless, it can not explain the postmortem redistribution of non-basic and non-lipophilic drugs. Moreover, the persistance of a drug metabolism immediately after death has to be considered. The examination of samples originating from different sites is of a great importance in order to 
repose sur le fait que les molécules lipophiles à large volume de distribution subissent d'importants phénomènes de redistribution. L'étude de la littérature montre cependant que cette explication est largement insuffisante pour de nombreuses molécules. D'autre part, l'éventualité de la poursuite du métabolisme de certaines molécules en période postmortem immédiate doit être envisagée. D'un point de vue pratique, il est impératif de pouvoir disposer de prélèvements autopsiques multiples afin de limiter les difficultés d'interprétation et d'approcher les mécanismes présidant à ces phénomènes de redistribution. Enfin, nous pensons qu'il est nécessaire de quantifier les variations observées, vraisemblablement sous forme de pourcentage, afin d'harmoniser l'interprétation de ces phénomènes.

\section{MOTS-CLÉS}

Redistribution post-mortem, organes réservoirs, lyse cellulaire, putréfaction, aspects pharmacocinétiques.

\section{Introduction}

En toxicologie médico-légale, la gravité ou le caractère mortel de l'intoxication sont généralement appréciés d'après la concentration sanguine du xénobiotique, pour laquelle existent souvent des valeurs de références (concentrations thérapeutiques, toxiques, létales). Mais alors que chez le vivant cette concentration sanguine permet d'évaluer, en tenant compte des caractéristiques pharmacocinétiques de la molécule, la quantité totale du xénobiotique présente dans l'organisme, ce raisonnement n'est plus toujours valable après le décès. En effet, les concentrations obtenues à partir des prélèvements réalisés en période post-mortem ne sont pas forcément le reflet de la concentration sanguine au moment du décès, en raison d'une part de variations des concentrations entre les différents sites de prélèvement, et d'autre part de variations de ces mêmes concentrations en fonction du délai écoulé entre le décès et les prélèvements. Ces phénomènes, regroupés sous le terme générique et quelque peu réducteur de redistribution post-mortem, rendent parfois délicate l'interprétation des résultats, d'autant plus que ce phénomène concerne des molécules très fréquemment rencontrées en toxicologie médico-légale, telles que les opiacés (1), les amphétamines (2), la cocaïne (3), ou les antidépresseurs tricycliques (4). La revue de la littérature montre que certains organes représentent des sources importantes de redistribution post-mortem. Ceci est cependant largement insuffisant pour expliquer certaines différences observées. Les modifications cellulaires et tissulaires liées aux phénomènes cadavériques ainsi que les caractéristiques pharmacocinétiques des molécules participent vraisemblablement avoid misinterpretations and to unserstand the underlying mechanisms of postmortem redistribution. Furthermore, the quantification of these site- and/or time-differences expression the form of ratios can be a way to harmonize the interpretation of the results.

\section{KEY-WORDS}

Postmortem redistribution, drug reservoirs, autolysis, putrefactive process, pharmacokinetics.

largement à ces phénomènes, mais la connaissance de ces mécanismes demeure encore limitée.

\section{Sources de redistribution}

Chez le vivant, les xénobiotiques se concentrent dans certains organes. Après le décès, les molécules présentes dans ces organes vont diffuser vers les structures anatomiques de voisinage. Ces organes, souvent qualifiés "d'organes réservoirs" peuvent être soit des organes creux tels que le tractus gastro-intestinal, soit des viscères à fort pouvoir de concentration tels que les poumons ou le foie. Le myocarde est également considéré comme un organe réservoir. La redistribution à partir de ces organes peut s'effectuer selon deux mécanismes distincts : diffusion par l'intermédiaire des structures vasculaires anatomiques, et diffusion de contiguiité, transpariétale, vers les différents organes de voisinage.

\section{$>$ Redistribution depuis le tractus digestif}

La plupart des molécules présentes dans l'estomac lors du décès peuvent se redistribuer vers les gros vaisseaux abdominaux et thoraciques et les organes de voisinage selon les deux mécanismes précédemment évoqués. Par voie anatomique, les molécules présentes dans l'estomac diffusent rapidement, par voie sanguine, vers l'aorte abdominale via le tronc cœliaque et l'artère mésentérique supérieure, et vers la veine cave inférieure via les veines hépatiques et la veine porte. La diffusion transpariétale vers les organes de voisinage affecte la base pulmonaire gauche, les cavités cardiaques gauches, le péricarde, le lobe hépatique gauche, la rate 
$(5,6)$, ainsi que le montre la figure 1 . Cette diffusion débute très précocement, puisque les concentrations d'amitriptyline et de fluoxétine commencent à s'élever dans le sang cardiaque dès la $2^{\mathrm{emc}}$ heure après le décès $(4,7)$. Pounder et Smith (5) ont largement décrit ce phénomène pour l'éthanol. Ceci a été également démontré pour l'amitriptyline, le méthanol et le lithium (8). En dernier lieu, la régurgitation de tout ou partie du contenu gastrique dans les voies aériennes peut entrâ̂ner le passage de certaines molécules, initialement contenues dans l'estomac, vers la circulation pulmonaire. Deux mécanismes - toujours précoces - semblent pouvoir être distingués : l'inhalation de tout ou partie du contenu gastrique lors du processus agonique (9) et la régurgitation passive du contenu gastrique dans les voies aériennes supérieures lors de la relaxation postmortem du sphincter œsophagien. Ce second phénomène est contemporain de l'apparition de la rigidité cadavérique (5), et serait favorisé par la manipulation du corps et la mise en décubitus dorsal (10). Il en résulte une élévation des concentrations dans les gros vaisseaux du médiastin, aorte et veine cave supérieure, plus marquée que celle observée au niveau des cavités cardiaques droites et gauches. Ceci a été clairement mis en évidence pour l'éthanol, le paracétamol et le dextro-

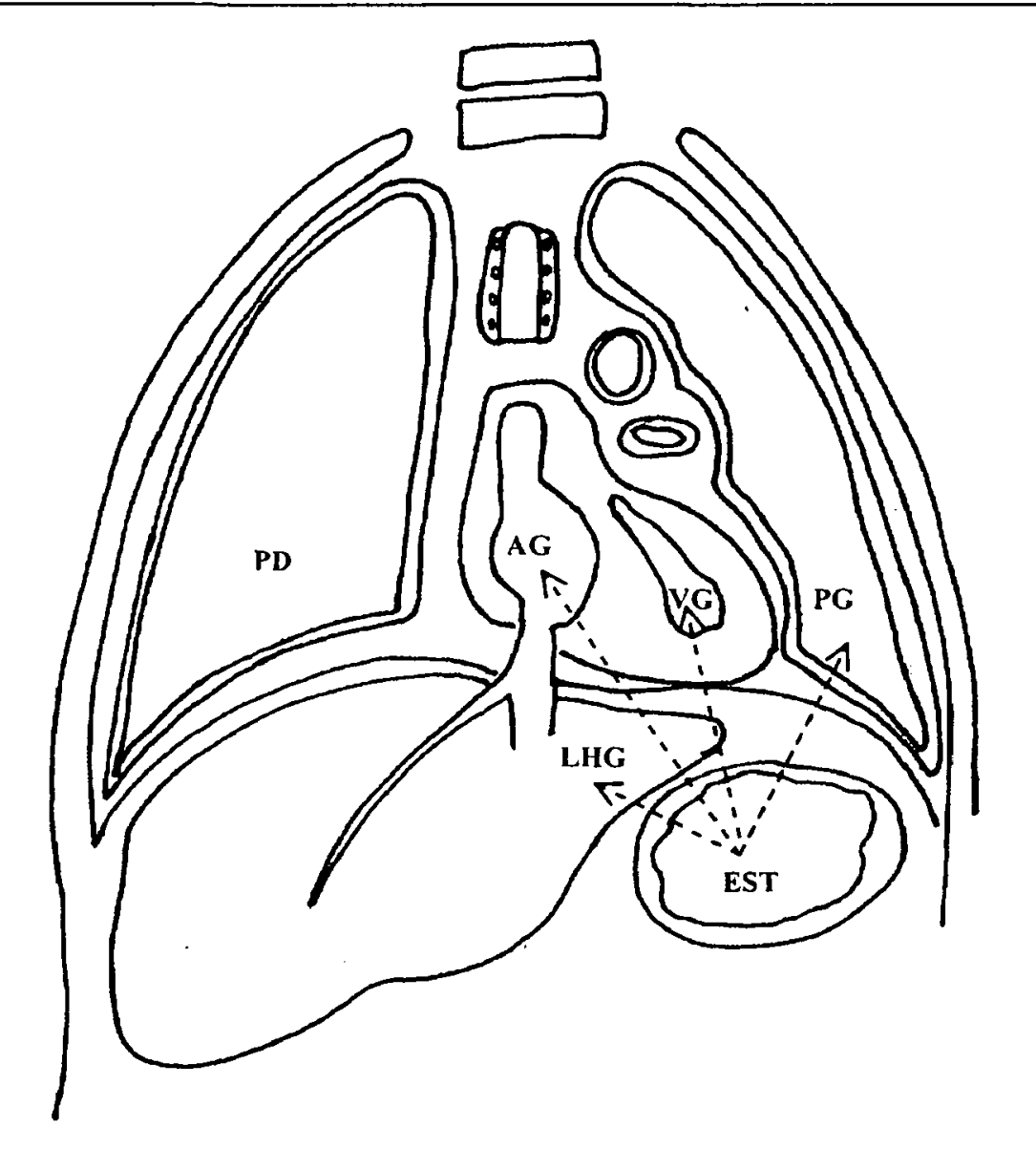

Figure 1 : Coupe frontale du thorax et de l'abdomen passant par l'atrium gauche montrant la redistribution postmortem précoce des molécules contenues dans l'estomac. les flèches indiquent le sens de la redistribution vers les principaux organes concernés (EST : estomac; LHG : lobe hépatique gauche; $P G$ : poumon gauche; $A G$ : atrium gauche; $V G$ : ventricule gauche; $P D$ : poumon droit). propoxyphène (11). Il semblerait d'autre part que ce phénomène de diffusion puisse intéresser également l'oesophage et l'intestin grêle (5). De manière générale, ce mécanisme de redistribution depuis le tractus gastro-intestinal est favorisé par le temps de contact du xénobiotique avec la muqueuse digestive, et il est ralenti par une conservation des corps à $4^{\circ} \mathrm{C}$ (5). Il est également influencé par l'état de réplétion de l'estomac (5). Enfin, l'existence de certaines pathologies pourrait influencer l'intensité de la redistribution. Ainsi Pounder et Smith (5) ont décrit une diminution de l'intensité de la redistribution depuis l'estomac vers le péricarde et les structures vasculaires du médiastin chez un sujet porteur d'un volumineux adénocarcinome de la base pulmonaire gauche, ainsi qu'une augmentation de l'intensité de cette redistribution chez un individu décédé d'un infarctus du myocarde.

\section{> Redistribution à partir des poumons}

Chez le vivant, les poumons reçoivent tout le flux sanguin issu du ventricule droit. Certaines molécules ont tendance à s'accumuler dans le parenchyme pulmonaire. Il s'agit essentiellement de bases faibles lipophiles, ayant un pKa proche de 8, telles que les antidépresseurs tricycliques, les amphétamines, la méthadone ou la chlorpromazine $(12,13,14)$. Le phénomène a également été décrit pour des molécules plus hydrosolubles telles que le méthanol (15). En post-mortem, la redistribution de ces molécules depuis les sites d'accumulation intra-pulmonaires vers la circulation pulmonaire est rapide et intense, et débute vraisemblablement dès la deuxième heure après le décès (16). Ce phénomène de diffusion entraîne une augmentation des concentrations dans les cavités cardiaques et les vaisseaux thoraciques (Figure 2), avec pour corollaire une diminution de concentration de ces molécules dans le parenchyme pulmonaire $(4,6,11)$. De nombreux cas d'augmentation des concentrations dans le sang des veines et artères pulmonaires ont ainsi été décrits dans la littérature, et font toujours mention d'une élévation des concentrations dans l'aorte et les cavités cardiaques gauches supérieure à celle des concentrations dans la veine cave supérieure et les cavités droites. Ceci a été rapporté en particulier pour l'amphétamine et la méthamphétamine (13) ou la lidocaïne (17). Les deux mécanismes précédemment décrits pour l'appareil digestif ont également été évoqués pour expliquer ce phénomène. I_e premier mécanisme évoqué est une redistribution par l'intermédiaire du réseau vasculaire pulmonaire : la paroi des capillaires veineux pulmonaires étant plus fine et perméable que celle des capillaires artériels, la redistribution à travers le réseau veineux pulmonaire serait donc supérieure à celle obser- 
vée à travers le réseau artériel $(10,18,19)$. Le second mécanisme est une redistribution vers les organes de contact. Il serait possible d'observer une diffusion directe, de contiguité, depuis le parenchyme ou les capillaires pulmonaires entourant les bronches gauches et la carina vers l'aorte et les cavités cardiaques gauches, puis plus tardivement vers la veine cave supérieure et les cavités droites (20). En effet, les concentrations aortiques sont supérieures à celles des cavités gauches, et la concentration dans la veine cave supérieure est supérieure à celle des cavités droites, laissant supposer que la diffusion vers ces vaisseaux se fait également directement par les tissus adjacents, et non pas uniquement par l'intermédiaire des vaisseaux pulmonaires (11). Selon Fuke et al. (6), ces phénomènes de diffusion à partir du tissu pulmonaire seraient plus intenses que ceux observés à partir de l'estomac. Ceci est vraisemblablement dû à l'importance et à la finesse de la surface d'échange, représentée en l'occurrence par les alvéoles pulmonaires, ainsi qu'à la grande richesse vasculaire du parenchyme pulmonaire (20). Ces deux phénomènes de diffusion peuvent naturellement coexister, et il s'avère difficile de déterminer leur part de responsabilité respective. Plusieurs auteurs ont tenté d'apporter des réponses. Selon Hilberg et al. (20), lorsque les concentrations dans le sang cardiaque sont supérieures à celles du tissu myocardique, le mécanisme prépondérant serait une diffusion depuis le paren-

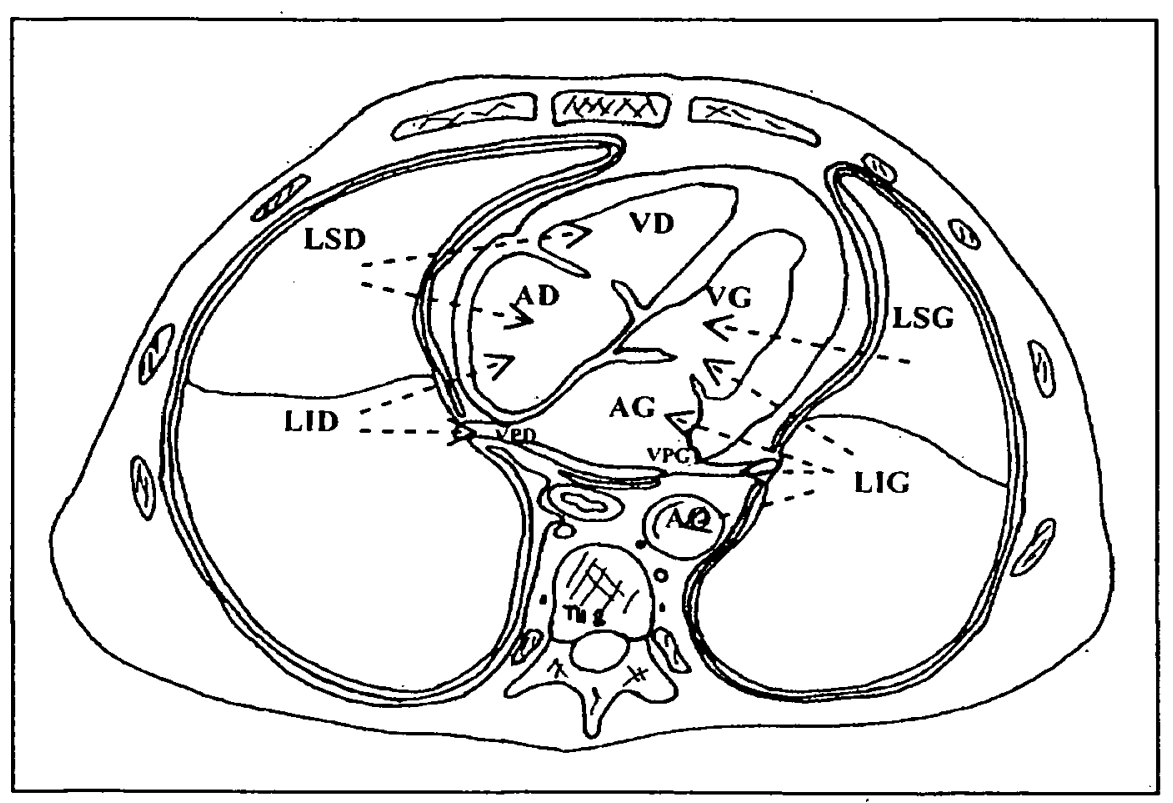

Figure 2 : Coupe transversale du thorax passant par la huitième vertèbre thoracique. Vue caudale. Les flèches indiquent le sens de la redistribution depuis le parenchyme pulmonaire vers les principaux organes concernés. La redistribution s'effectue par voie vasculaire par l'intermédiaire des veines pulmonaires (VPG : veine pulmonaire gauche; $V P D$ : veine pulmonaire droite) et par voie transpariétale vers les structures anatomiques de voisinage ( $A G$ : atrium gauche; VG : ventricule gauche; $A O$ : aorte ; $L S G$ : lobe supérieur du poumon gauche; LIG : lobe inférieur du poumon gauche ; LSD : lobe supérieur du poumon droit ; $L I D$ : lobe inférieur du poumon droit ; $A D$ : atrium droit ; $V D$ : ventricule droit). chyme pulmonaire via les vaisseaux pulmonaires, alors que, lorsque les concentrations dans le tissu myocardique sont supérieures à celles retrouvées au niveau du sang cardiaque, le mécanisme prépondérant serait soit une diffusion directe depuis l'estomac, soit une forte concentration dans les cellules myocardiques (cf. infra). De même, selon Pounder (10), lorsque les concentrations dans le sang cardiaque sont supérieures à celles du liquide péricardique, il s'agirait très vraisemblablement d'une diffusion depuis le parenchyme pulmonaire, l'inverse traduisant une diffusion depuis l'estomac. Enfin, une diffusion pourrait s'effectuer des poumons vers le foie vraisemblablement par l'intermédiaire des liquides pleural et péritonéal qui s'écouleraient à travers le diaphragme (16). Fuke et al. (6) ont confirmé ces résultats en démontrant une élévation des concentrations en toluène dans le lobe hépatique gauche après instillation dans les voies aériennes supérieures.

\section{$>$ Redistribution à partir du foie}

Les phénomènes de redistribution au niveau du foie sont extrêmement complexes parce qu'ils font appel à des mécanismes multiples. Le foie représente en effet une source majeure de redistribution, soit par l'intermédiaire de la circulation hépatique, soit par diffusion vers les organes de voisinage. Par ailleurs, il peut être lui-même la cible d'un phénomène de redistribution, en particulier des molécules présentes dans le tractus gastro-intestinal lors du décès. Les xénobiotiques concentrés dans le parenchyme hépatique au moment du décès peuvent passer dans la circulation cave inférieure par le système porte. De là, ils peuvent diffuser de proche en proche vers les cavités cardiaques droites et les vaisseaux pulmonaires. Une redistribution vers le sang veineux périphérique, bien que mineure, a également été décrite, à partir de la veine cave inférieure (21). Il résulte de cette redistribution une diminution des concentrations des molécules dans les deux lobes hépatiques. C'est ainsi que Pohland et Bernhard (7) notent une diminution des concentrations hépatiques de fluoxétine et norfluoxétine de $39 \%$ et $23 \%$, respectivement, entre la $2^{\text {eme }}$ et la $12^{\text {zmc }}$ heure post-mortem. Ce phénomène de diffusion serait cependant plus long et moins intense que la redistribution à partir des poumons (21). D'autre part, la redistribution des xénobiotiques concentrés dans le parenchyme hépatique avant le décès peut se faire par diffusion de contiguité vers les organes de voisinage, en l'occurrence l'œsophage, l'estomac, le pylore, le duodénum proximal et la vésicule biliaire, mais il semble que ceci soit de peu d'importance au regard des autres phénomènes de redistribution post-mortem. Enfin, compte tenu des rapports anatomiques étroits 
entre le foie et l'estomac, les xénobiotiques présents dans l'estomac lors du décès peuvent pénétrer le parenchyme hépatique soit par diffusion passive, soit par l'intermédiaire de la circulation veineuse hépatique (21). Ce phénomène de diffusion est très inégal, le lobe hépatique gauche, au contact de l'estomac, étant naturellement plus concerné que le lobe hépatique droit (Figure 3). C'est ainsi que Pounder et Davis (22) ont mis en évidence des concentrations nettement plus élevées de zopiclone dans le lobe hépatique gauche et la vésicule biliaire que dans le lobe hépatique droit. Fuke et al. (6) ont constaté le même phénomène après instillation de toluène dans l'estomac. Il est donc particulièrement difficile d'interpréter correctement l'évolution des concentrations hépatiques en période post-mortem. Selon Hilberg et al. (23), le pH des molécules pourrait intervenir dans ces différents mécanismes. Enfin, les phénomènes de redistribution impliquant le parenchyme hépatique concernent un très grand nombre de molécules dont il est impossible d'établir une liste exhaustive.

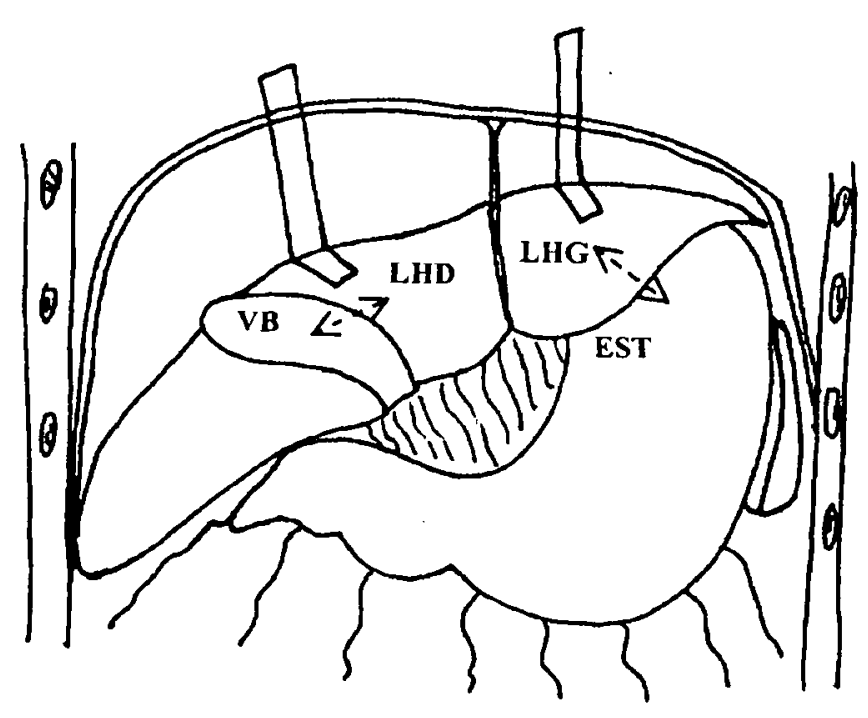

Figure 3 : Vue antérieure de l'abdomen, après ouverture des plans cutanés et du péritoine pariétal antérieur, montrant la redistribution depuis le parenchyme hépatique. Les doubles flèches indiquent que la redistribution peut s'effectuer dans les deux sens. ( $L H G$ : lobe hépatique gauche; $L H D$ : lobe hépatique droit ; VB : vésicule biliaire ; EST: estomac).

\section{$>$ Redistribution à partir du myocarde}

Certains médicaments, généralement à visée cardiologique, tels que les digitaliques ou les inhibiteurs calciques, mais aussi d'autres molécules telles que les quinidiniques, se concentrent dans le myocarde, avec un rapport de concentrations myocarde/plasma parfois très élevé (de l'ordre de 30/1 pour la digoxine). Lors du décès, ces molécules sont relarguées dans le sang cardiaque où leur concentration s'élève alors considérablement. Une augmentation plus modérée mais constante est également retrouvée au niveau de la veine sous-clavière, ce qui est un argument pour refuser ce site comme site de prélèvement sanguin périphérique (24). Ce mécanisme a également été mis en évidence pour d'autres molécules, telles que la morphine (25, 26), l'amphétamine $(24,27)$, la méthamphétamine $(13$, 27), le propoxyphène et le norpropoxyphène (24), l'imipramine et la désipramine (28), l'amitriptyline, la doxépine, la maprotiline ou le métoprolol (24). Cependant, il est là encore parfois difficile de distinguer une diffusion depuis le myocarde d'une diffusion vers le myocarde, à partir du parenchyme pulmonaire ou de l'estomac. Le sang cardiaque peut donc faire l'objet d'une redistribution de molécules provenant de l'estomac, du parenchyme pulmonaire, du parenchyme hépatique et enfin du myocarde lui-même.

\section{$>$ Redistribution vers les tissus adipeux}

A l'inverse des phénomènes précédemment décrits, certains xénobiotiques présents dans la circulation générale lors du décès peuvent secondairement être redistribués vers certains tissus ou organes, entraînant une diminution des taux plasmatiques du xénobiotique. Ainsi a-t-on déjà évoqué ce phénomène pour le foie. Les tissus graisseux font également l'objet de ce phénomène. Des molécules très lipophiles peuvent continuer à diffuser vers les tissus lipidiques en période post-mortem. En effet, la vascularisation de ces tissus étant faible, le décès toxique survient généralement avant l'équilibre des concentrations entre le sang et le tissu graisseux. Ce phénomène est susceptible de concerner les antidépresseurs tricycliques, les anesthésiques et surtout les volatils (6). Il en résulte une diminution de la concentration sanguine du xénobiotique, avec pour corollaire une augmentation des concentrations tissulaires.

\section{Phénomènes agoniques et cadavériques \\ $>$ Lyse cellulaire}

Le mécanisme le plus souvent évoqué par le passé pour expliquer le relargage dans le secteur plasmatique de molécules contenues à l'intérieur des cellules, en particulier de molécules basiques lipophiles, est l'acidification du $\mathrm{pH}$ sanguin qui entraînerait un retour des composés basiques vers ce secteur (21). En réalité, il s'agit très vraisemblablement d'un mécanisme beaucoup plus complexe, d'autant plus qu'il paraît clair à présent que le $\mathrm{pH}$ cellulaire diminue avant le $\mathrm{pH}$ plasmatique. En effet, l'hypoxie contemporaine des phénomènes ago- 
niques entraîne un épuisement de la production d'ATP, avec poursuite du métabolisme en anaérobiose (29). Ceci a pour corollaire la production d'acide pyruvique et lactique, entraînant une baisse du pH cellulaire, donc vraisemblablement à ce stade une augmentation du transfert des molécules basiques vers l'intérieur des cellules. La diminution de la quantité d'ATP disponible entraîne ensuite un dysfonctionnement des pompes $\mathrm{Na}^{+} / \mathrm{K}^{+}$ATP-dépendantes, avec équilibration des concentrations en ions $\mathrm{Na}^{+}$et $\mathrm{K}^{+}$entre les milieux intraet extracellulaires, le $\mathrm{pH}$ de ce dernier diminuant à son tour. Parallèlement, l'eau présente dans le secteur extracellulaire pénètre à l'intérieur de la cellule. Enfin, cette interruption de la synthèse d'ATP entraîne des altérations des mitochondries et du réticulum endoplasmique. Ces organites vont alors libérer à l'intérieur du cytoplasme d'importantes quantités de calcium, qui vont activer phospholipases et protéases. Ces enzymes vont à leur tour dégrader la membrane cellulaire. En dernier lieu, la chute du $\mathrm{pH}$ et l'altération des lysosomes entraînent la libération dans le cytoplasme des enzymes lysosomales qui, activées, vont digérer les différents organites cellulaires (29). L'ensemble de ces phénomènes aboutit à la lyse de la membrane cellulaire et à la libération dans le milieu extracellulaire d'enzymes - hépatiques et cardiaques notamment - et d'acides aminés initialement présents dans la cellule. Langford et Pounder (30) ont ainsi proposé d'utiliser comme marqueurs de la redistribution pulmonaire le relargage dans les vaisseaux pulmonaires d'acides aminés tels que la glycine, la leucine, la méthionine, la sérine et la valine. Les macromolécules présentes dans la cellule sont également libérées dans le milieu extracellulaire. De la même manière, les xénobiotiques basiques lipophiles, fortement concentrés à l'intérieur des cellules, vont passer dans le secteur interstitiel puis plasmatique. Les molécules plus acides ou neutres, moins concentrées dảns les cellules avant le décès, sont donc théoriquement moins affectées par ces phénomènes de redistribution. Ces phénomènes de lyse atteignent toutes les cellules de l'organisme, mais avec une vitesse variable. Les altérations des cellules de l'intima des vaisseaux participent également aux phénomènes de redistribution. Skopp et al. (31) ont mis au point un modèle in vitro destiné à évaluer l'intensité de la perméation vasculaire dans les 120 heures suivant le décès. Ils ont ainsi démontré que, durant cette période, la diffusion des molécules depuis la lumière vasculaire vers le secteur extravasculaire augmentait selon une courbe sigmoïde, et que ce processus était quantitativement supérieur au processus inverse de diffusion depuis le secteur extravasculaire vers la lumière du vaisseau. Toutefois, les conditions expérimentales de cette étude sont très différentes de celles observées sur le cadavre, le $\mathrm{pH}$ sanguin, en particulier, ayant été ajusté à 7,4 durant toute la durée de l'expérimentation. Or l'intensité de ce processus de perméation serait particulièrement dépendante de l'acidification du $\mathrm{pH}$ plasmatique. La température ambiante, le gradient de concentration de la molécule de part et d'autre de la paroi du vaisseau, ainsi que la taille, la charge et la conformation de la molécule interviendraient également. Enfin, la conservation du corps au froid minimiserait ce processus pendant les 48 heures qui suivent le décès. Au-delà, ce processus de perméation vasculaire augmente, quelle que soit la température du corps (31).

\section{$>$ Phénomènes de coagulation et d'hypo- stase}

Après le décès, le sang sédimente et coagule de manière inégale dans l'organisme. Ce phénomène de coagulation, très variable d'un site anatomique à l'autre, en fonction notamment de la position du cadavre, est suivi d'une lyse du caillot. Au mieux, celle-ci peut aboutir à un sang laqué complètement fluide et incoagulable (21). D'autre part, les phénomènes d'hypostase, contemporains de l'installation des lividités cadavériques et débutant 3 à 5 heures après le décès, entrâ̂nent des variations considérables de l'hématocrite entre les différentes régions anatomiques (31). Thomsen et al. (32) ont ainsi démontré que l'hématocrite diminuait de plus de $50 \%$ dans les 9 heures suivant le décès, pour des prélèvements effectués en région veineuse cubitale. La position des cadavres n'était malheureusement pas précisée dans cette étude. Ces phénomènes sont susceptibles d'affecter les concentrations de molécules présentant une fixation érythrocytaire importante (cf. infra).

\section{$>$ Mouvements entre les différents com- partiments sanguins}

La redistribution des xénobiotiques depuis les organes solides vers le sang n'est pas uniquement due à un phénomène de diffusion passive. Des mouvements sanguins, survenant au cours de l'évolution des phénomènes cadavériques, et essentiellement dus à des modifications de pression et de fluidité, interviennent également. Au cours des 24 premières heures après le décès, la rigidité cadavérique, qui touche les muscles lisses comme les muscles striés, entraîne une contraction des ventricules en systole. La rigidité cardiaque est précoce, puisqu'elle débute généralement dans les deux premières heures après le décès (33). Ce phénomène entraîne le reflux d'une faible quantité de sang cardiaque vers la veine cave supérieure et les veines sousclavières. Puis, l'augmentation de la pression intra- 
abdominale entraîne un reflux de sang de l'aorte abdominale vers l'aorte thoracique, et de la veine cave inférieure vers l'oreillette droite et les veines pulmonaires. Lors de l'apparition de la putréfaction, la rigidité disparaît progressivement. Les gaz de putréfaction intestinale distendent l'abdomen et repoussent le diaphragme. Le sang, refoulé des viscères, gagne les membres, les parois abdominales et thoraciques, rendant ainsi apparent le trajet des veines superficielles. Ce phénomène, improprement qualifié de "circulation posthume", puisqu'il ne s'agit pas d'une circulation sanguine stricto sensu, tendrait à égaliser les concentrations des toxiques entre les différents compartiments (24). Il est toutefois important de noter que ces phénomènes sont continus, mais vraisemblablement de très faible importance et variables d'un cadavre à un autre. Enfin, la seule étude réalisée sur cadavre humain date de 1961 (34) et mériterait d'être réactualisée. Gomez-Zapata et al. (35) décrivent des mécanismes voisins chez le lapin.

\section{$>$ Phénomènes putréfactifs}

La putréfaction, dont l'apparition est éminemment variable en fonction des conditions climatiques et de l'état du cadavre, est marquée par une prolifération bactérienne susceptible d'induire une dégradation et/ou une néoformation de certains xénobiotiques. La synthèse et la dégradation de l'éthanol en représentent l'exemple le plus flagrant. Les bactéries normalement présentes dans le tube digestif au moment du décès envahissent le système veineux portal et les vaisseaux lymphatiques intestinaux (36). Ce mécanisme débute dans les premières heures après le décès, d'autant plus rapidement d'ailleurs que le cadavre est placé dans une atmosphère dont la température est élevée (37). Les bactéries concernées sont essentiellement de Bacillus spp., Pseudomonas spp., Escherichia coli, Proteus mirabilis, Clostridium perfringens, Staphylococcus epidermidis, Streptococcus facalis et Bacteroides fragilis. La prolifération microbienne et la rupture des membranes anatomiques généralisent ensuite ce processus, qui gagne de proche en proche les différents organes et tissus (38). Ces bactéries, en présence de différents substrats, essentiellement glucidiques ou protidiques, sont susceptibles de dégrader les xénobiotiques, voire d'en synthétiser certains. Le problème la production et/ou de la dégradation de l'éthanol par les micro-organismes est particulièrement épineux. En présence de glucose, lactate, glycérol, ou encore d'acides aminés provenant de la dégradation des protéines, certains micro-organismes synthétisent de l'éthanol (Corry, 1978). Selon O'Neal et Poklis (36), il s'agit essentiellement de Candida albicans et Escherichia coli, mais une cinquantaine de bactéries et une vingtaine de levures seraient en fait capables de synthétiser de l'éthanol. Plus récemment, Kupfer et al. (39) ont mis au point une technique d'amplification par PCR permettant l'identification rapide de Candida albicans, Escherichia coli et Proteus vulgaris dans des échantillons sanguins post-mortem, ceci afin d'apporter un argument supplémentaire à l'hypothèse d'une néoformation d'éthanol lorsque l'interprétation des résultats s'avère difficile. $D$ ' autre part, le glucose étant le principal substrat utilisé, les organes les plus riches en glucose seraient ainsi le siège d'une néoformation accrue d'éthanol. Il s'agit naturellement du foie, mais aussi des muscles squelettiques, du myocarde et des poumons, des urines d'individus diabétiques, alors que le cerveau ne serait concerné qu'à un moindre degré $(36,40)$. Il en résulte d'importantes variations des concentrations d'éthanol entre les différents sites de prélèvement (41). D'autre part, la production d'éthanol post-mortem s'accompagne fréquemment de la production d'autres alcools, en particulier de méthanol, de npropanol, d'isopropanol, de n-butanol et de sec-butanol (42) dont la présence pourrait servir de marqueur de la néoformation d'éthanol $(43,44)$. Enfin, selon Jones et al. (45), l'administration de mannitol avant le décès favoriserait cette néoformation. Ces phénomènes ont amené les différents auteurs à proposer l'humeur vitrée, milieu très protégé des contaminations bactériennes, comme milieu alternatif pour distinguer en particulier une formation endogène d'éthanol d'un apport exogène ante-mortem (cf.infra). L'éthanol peut également subir une dégradation sous l'action de certains micro-organismes par oxydation en acétaldéhyde puis en acétate. Ces phénomènes sont moins bien connus que les précédents. Certains micro-organismes semblent capables de synthétiser aussi bien que de dégrader l'éthanol; il s'agit de levures et de bactéries à pouvoir fermentatif ou oxydatif - selon les orientations métaboliques du milieu - telles que Candida albicans ou Serratia marcescens (46), alors que d'autres microorganismes, à métabolisme strictement oxydatif, ne peuvent induire qu'une dégradation de l'éthanol. C'est notamment le cas de Pseudomonas spp. (38). La chronologie de ces phénomènes est controversée. Selon Takayasu et al. (47), qui ont administré de l'éthanol deutéré per os ou IV à différents lots d'animaux, la concentration d'éthanol deutéré décroît progressivement dans les différents organes au cours des premières heures après le décès, alors qu'augmente progressivement la concentration d'éthanol non deutéré et de 1propanol, suggérant ainsi que l'étape de dégradation précède l'étape de néoformation. Selon Bouillerot et Laviano-Rousselin (38), au contraire, la dégradation de l'éthanol n'interviendrait qu'après une étape de synthèse en fonction de l'épuisement des substrats dispo- 
nibles. Ce phénomène de dégradation, particulièrement documenté pour l'éthanol, peut également concerner d'autres molécules. Les benzodiazépines en sont un bon exemple. Robertson et Drummer (48) ont ainsi montré que les nitrobenzodiazépines (flunitrazépam, clonazépam, nitrazépam) étaient progressivement dégradées en leur métabolites respectifs au niveau des poumons, du cœur, du foie, des reins et des muscles squelettiques sous l'action de diverses bactéries possédant une nitroréductase (Escherichia coli, Bacillus spp., Proteus mirabilis, Clostridium perfringens, Staphyloccocus aureus, Staphyloccocus epidermidis, Streptoccocus facalis et Bacteroides fragilis). Selon ces mêmes auteurs, ces phénomènes sont inhibés par la conservation des corps à $4^{\circ} \mathrm{C}$. Il semblerait enfin que les ions cyanures subissent également un phénomène de dégradation sous l'influence de certaines bactéries. Ballante et al. (49) ont décrit une diminution significative de la concentration en ions cyanures dans le sang et les tissus d'animaux euthanasiés avec de l'acide cyanhydrique à différents temps de prélèvements. La principale hypothèse évoquée est une transformation des ions cyanures en thiocyanates sous l'action des bactéries de la putréfaction. Les travaux concernant la redistribution des ions cyanures sont cependant très anciens et peu nombreux et doivent nécessairement faire l'objet de nouvelles études visant notamment à déterminer quels sont les micro-organismes responsables. La putréfaction est donc susceptible d'engendrer dans un premier temps des variations des concentrations entre les différents sites et les différents temps de prélèvement. A un stade plus avancé, lorsque les viscères sont détruits, on observe au contraire une tendance à l'égalisation des concentrations dans les différents sites de prélèvement.

\section{Aspects pharmacocinétiques}

A côté des phénomènes physico-chimiques liés à la dégradation des différentes cellules en période postmortem, les caractéristiques pharmacocinétiques des xénobiotiques interviennent également dans l'intensité de leur redistribution. Il est important de noter en préambule à ce paragraphe que les concentrations d'une même molécule peuvent varier in vivo entre les différents sites et les différents temps de prélèvement. En effet, la concentration artérielle d'un grand nombre de molécules est supérieure à la concentration veineuse pendant les phases d'absorption et de distribution. Ceci a bien été décrit pour l'éthanol, le diazépam, le phénobarbital dans les minutes qui suivent leur administration, et ceci quelles qu'en soient les modalités $(24,50)$. Ces différences sont d'autant plus marquées que les molécules ont une demi-vie courte (50). Pendant la phase d'élimination, les concentrations de certaines molécules (furosémide, procainnamide, propanolol) seraient plus élevées dans le sang veineux que dans le sang artériel (50). Il est donc possible que la distribution des molécules entre les différents secteurs immédiatement avant le décès puisse influer sur la redistribution ultérieure de la molécule concernée. Ceci a été évoqué par Elsirafy et al. (51) pour expliquer les différences de concentration hépatique du diazinon administré simultanément à un lot d'animaux morts et à un autre lot d'animaux euthanasiés secondairement à différents temps. Les différentes étapes de la cinétique d'une molécule dans l'organisme sont l'absorption, la distribution, le métabolisme et l'élimination. La redistribution d'une molécule est vraisemblablement conditionnée à la fois par ses propres caractéristiques pharmacocinétiques et par les modifications post-mortem des cellules et des tissus. Les seules études consacrées à ce sujet tendent à caractériser l'intensité de la redistribution d'une molécule uniquement à partir de sa distribution tissulaire in vivo, donc à partir de son volume de distribution. Le phénomène est certainement beaucoup plus complexe, avec notamment l'éventualité de la persistance pendant quelques heures du métabolisme de certaines molécules. Il est probable qu'à toutes les étapes de la cinétique d'une molécule puissent intervenir des modifications dans la période post-mortem immédiate, modifications susceptibles de majorer les phénomènes de redistribution.

\section{$>$ Absorption}

L'absorption d'un xénobiotique au travers des membranes de l'organisme peut se dérouler selon différentes modalités, la plus répandue étant la diffusion passive. Cette diffusion, régie par la loi de Fick, fait intervenir entre autres paramètres la taille, la lipophilie et l'état d'ionisation de la molécule, mais aussi le $\mathrm{pH}$ du milieu, la surface et l'épaisseur de la membrane. Bien qu'aucune étude n'ait été à notre connaissance consacrée à ce sujet, il est très probable que la diffusion soit altérée par les modifications physico-chimiques précédemment décrites, en particulier par l'acidification du $\mathrm{pH}$ cellulaire et plasmatique et par la perte de cohésion de la structure membranaire. Or la majorité des xénobiotiques absorbés par voie orale traversent la muqueuse digestive selon ce mécanisme (acides faibles au niveau de l'estomac, bases faibles au niveau intestinal). Il est donc possible que l'absorption des xénobiotiques présents dans le tube digestif au moment du décès soit modifiée. Il faudrait d'autre part, pour interpréter correctement ces mouvements de diffusion, connaître l'évolution du $\mathrm{pH}$ des différents segments du 
tractus digestif en période post-mortem immédiate. La filtration est un autre mécanisme d'absorption concernant essentiellement les petites molécules hydrosolubles qui suivent généralement le flux d'eau à travers des pores protéiques, selon un gradient de pression osmotique ou oncotique. C'est le cas notamment de l'éthanol. L'égalisation des concentrations en eau et électrolytes de part et d'autre des membranes cellulaires et l'altération des protéines membranaires tend très vraisemblablement à accélérer puis stopper ce processus de filtration, une fois l'équilibre atteint. Le troisième mécanisme d'absorption fréquemment rencontré est le transport actif. Ce mécanisme concerne surtout les acides et bases faibles organiques. Il nécessite un transporteur protéique et s'effectue toujours contre un gradient de concentration. Il nécessite donc de l'énergie, fournie par l'ATP. L'arrêt précoce de la synthèse d'ATP bloque donc ce mécanisme de transfert, quelques minutes à quelques heures après le décès.

\section{$>$ Distribution}

Quelle que soit la voie d'absorption, les xénobiotiques atteignent ensuite le compartiment sanguin pour être distribués vers les différents liquides de l'organisme, puis vers les tissus solides.

\section{Transport sanguin des xénobiotiques}

La première étape de la distribution est le transport sanguin. Dans le sang, les xénobiotiques peuvent (i) se dissoudre dans l'eau plasmatique; (ii) se lier - dans des proportions variables - aux protéines plasmatiques; (iii) se lier à la membrane cellulaire ou pénétrer dans le cytoplasme des éléments figurés du sang, des érythrocytes en particulier. Seules les molécules les plus hydrosolubles peuvent se dissoudre dans l'eau plasmatique. C'est le cas en particulier de l'éthanol, qui diffuse très rapidement dans l'ensemble du compartiment hydrique de l'organisme, et dont la redistribution n'est pas influencée par les variations post-mortem du transport sanguin. La liaison aux protéines plasmatiques peut se faire selon différentes modalités. Il peut s'agir soit de liaisons hydrophobes faibles entre des toxiques liposolubles et les sites hydrophobes de l'albumine et des lipoprotéines (buprénorphine, par exemple), soit de liaisons électrostatiques entre des acides faibles ionisés et les sites cationiques de l'albumine ou entre les bases faibles ionisées et les sites anioniques de l' $\alpha 1$-glycoprotéine acide (exemple : $\beta$-bloquants, sulfamideshypoglycémiants). Des liaisons de covalence irréversibles entre l'albumine et certaines molécules (exemple : antipaludéens de synthèse) sont également décrites (52). Or le taux de protéines plasmatiques diminue après le décès. Plusieurs hypothèses ont été envisagées. Selon Konikova et al. (53), les protéines plasmatiques sont dégradées en acides aminés dans le sang circulant et dans les tissus rapidement après le décès, vraisemblablement sous l'action conjuguée des phénomènes anoxiques et de la libération des enzymes protéolytiques dans le secteur vasculaire. Selon Bonte et al. (54), ce processus s'accélérerait lors de la putréfaction sous l'action des bactéries protéolytiques. Concernant plus spécifiquement l'albumine, Oemichen et al. (55) ont suggéré que la diminution de l'albumine dans le secteur vasculaire durant les 48 premières heures suivant le décès pourrait être due à un phénomène de redistribution vers le secteur périvasculaire et les tissus environnants. Même si ces travaux sont anciens et très incomplets, il semble clair que la fixation protéique est diminuée en période post-mortem, augmentant ainsi la fraction libre active dans le secteur vasculaire, phénomène qui, de manière théorique, devrait tendre à diminuer l'intensité de la redistribution de ces molécules. D'autre part, nous n'avons pas retrouvé d'informations dans la littérature quant au devenir post-mortem de l' $\alpha 1$-glycoprotéine acide ou des lipoprotéines. De toutes manières, le fait que les dosages en toxicologie soient toujours effectués sur sang total ou sur sérum sans filtration minimise considérablement la portée de ce phénomène. La fixation érythrocytaire est un phénomène mineur en comparaison du précédent. Cette fixation ne concerne qu'un nombre relativement limité de molécules. La concentration de ces molécules dans le sang total peut être affectée par les phénomènes de coagulation puis de lyse survenant après le décès, mais surtout par les phénomènes d'hypostase entraînant le passage de plasma et d'éléments figurés du sang vers le secteur extravasculaire, avec des variations importantes de l'hématocrite entre les différents sites anatomiques (1). Ce phénomène a été évoqué par Tomson et al. (56) au sujet de la redistribution post-mortem de la phénytoïne ou par Skopp et al. (57) au sujet de celle des dérivés glucuronoconjugués de la morphine. Ce phénomène est cependant insuffisant pour expliquer à lui seul les différences de concentration observées dans certains cas (58).

\section{Distribution tissulaire}

In vivo, la distribution tissulaire est un phénomène dynamique qui dépend des propriétés physico-chimiques des molécules, mais aussi de facteurs physiologiques tels que les débits sanguins locaux et l'affinité particulière de certains xénobiotiques pour des composés endogènes spécifiques. Les caractéristiques physico-chimiques des molécules sont un élément déterminant de l'intensité de leur redistribution post-mortem. 
Les molécules présentes dans le plasma pénètrent dans les cellules essentiellement par transfert passif ou filtration à travers les membranes lipidiques. Seules les formes libres non ionisées de ces molécules ont une liposolubilité suffisante pour diffuser du liquide plasmatique vers le liquide interstitiel, puis vers le liquide intracellulaire. Compte tenu du fait que le $\mathrm{pH}$ intracellulaire est plus acide que le $\mathrm{pH}$ plasmatique, les bases faibles sont plus volontiers sous forme ionisée dans la cellule que dans le plasma, ce qui facilite leur accumulation dans le secteur intracellulaire. C'est le phénomène de piégeage d'ions (ion trapping). Ce phénomène a été particulièrement bien étudié pour le parenchyme pulmonaire. Les molécules lipophiles basiques auraient ainsi tendance à s'accumuler dans les mitochondries et les lysosomes (12) dont sont particulièrement riches les macrophages alvéolaires (14), et pourraient être libérées en grandes quantités lors de la lyse cellulaire. La principale grandeur pharmacocinétique caractérisant l'intensité de cette distribution tissulaire est le volume apparent de distribution (Vd). Il s'agit en fait du volume fictif dans lequel se répartirait la molécule pour obtenir les concentrations plasmatiques observées. Ce volume de distribution ne reflète en rien la répartition des molécules dans les différents organes et n'est qu'un indicateur global de l'intensité de la distribution. Un très grand nombre de travaux tendent à démontrer que les molécules à grand $\mathrm{Vd}$ sont celles qui subissent les phénomènes de redistribution post-mortem les plus intenses, du fait de leur stockage tissulaire (59). Il s'agit le plus souvent de bases faibles lipophiles pour les raisons précédemment évoquées. Ceci a été très largement démontré pour les antidépresseurs tricycliques tels que l'amitriptyline (4), la nortriptyline (19), la trimipramine $(60)$, la dothiépine $(61,62)$. Des résultats similaires ont été démontrés pour d'autres molécules à large $\mathrm{Vd}$ telles que la fluoxétine (7), la venlafaxine (63), la méthadone $(64,65)$, l'amphétamine et la méthamphétamine (13), ou encore la digoxine (66). En fait, selon Hilberg et al. (67), seraient susceptibles d'être concernées toutes les molécules basiques lipophiles dont le Vd est égal ou supérieur à $3 \mathrm{~L} / \mathrm{Kg}$. La situation est beaucoup plus complexe pour d'autres molécules, ce qui doit nous amener à nuancer les affirmations précédentes. Le cas de la morphine et de ses dérivés glucuronoconjugués est particulièrement intéressant. Selon Sawyer et Forney (25), qui ont étudié la redistribution de la morphine libre et totale chez le rat, ces concentrations augmentent d'une part en fonction des sites de prélèvement - le foie et le myocarde étant les sites présentant les concentrations les plus élevées mais aussi en fonction du délai entre le décès et le prélèvement, ce second résultat ayant été infirmé par des études ultérieures $(26,68)$ selon lesquelles les taux de morphine restent stables au cours du temps, même s'il existe des variations des concentrations entre les différents sites de prélèvement, les concentrations dans le compartiment central étant plus élevées que les concentrations dans le compartiment périphérique. La majorité des auteurs s'accorde donc à reconnaître que les concentrations en morphine libre et conjuguée varient significativement entre les sites de prélèvement, indiquant la possibilité d'une redistribution. Or la morphine n'est pas une base faible, mais plutôt une molécule amphotère, dont la lipophilie varie avec le $\mathrm{pH}$, et dont le volume de distribution se situe aux alentours de $3 \mathrm{~L} / \mathrm{Kg}$. Les dérivés glucuroconjugués ont quant à eux un Vd faible, de l'ordre de $0,28 \mathrm{~L} / \mathrm{Kg}$. En ce qui concerne la redistribution de la morphine, les hypothèses sont multiples. Selon Sawyer et Forney, (25) l'abaissement du $\mathrm{pH}$ post-mortem diminuerait la lipophilie de la molécule, qui serait ainsi rapidement relarguée dans le secteur vasculaire. Selon Skopp et al. (1), les phénomènes d'hypostase, entraînant des variations de la teneur en eau et en érythrocytes dans les différents secteurs du compartiment vasculaire, pourraient intervenir dans cette redistribution. Ceci est toutefois insuffisant pour expliquer les variations de concentration observées entre différents organes dans les études précédentes. La variation des concentrations entre les sites des dérivés glucuronoconjugués pourrait être due, selon Carrupt et al. (69), au fait que ces molécules peuvent coexister dans l'organisme sous deux conformations différentes, l'une de ces conformations, en l'occurrence la forme dite "repliée", étant plus lipophile que l'autre. Enfin, fait particulièrement intéressant, la redistribution de la morphine est concentration-dépendante, avec accentuation des variations, en particulier entre le sang cardiaque et le sang veineux fémoral, aux doses toxiques par rapport aux doses thérapeutiques (25). Par ailleurs, Gomez et al. (70) décrivent chez l'animal une redistribution importante du paracétamol, molécule faiblement liposoluble, avec un volume de distribution de l'ordre de $1 \mathrm{~L} / \mathrm{Kg}$. Cette redistribution se caractérise par une augmentation lors des différents temps de prélèvement des concentrations sanguines dans les sites centraux (cavités cardiaques et veine cave inférieure), alors que les sites périphériques sont épargnés. Deux hypothèses sont avancées par les auteurs pour expliquer ce phénomène : (i) la possibilité d'une diffusion depuis l'estomac vers la région cardiaque, le paracétamol ayant été administré par voie orale, (ii) la possibilité d'une redistribution à partir de certains organes concentrant sélectivement le paracétamol, en l'occurrence le foie. Il aurait été intéressant, afin de tester ces hypothèses, de réaliser la même expérimentation en administrant le paracétamol par voie 
parentérale, et surtout de disposer de prélèvements de parenchyme hépatique, à différents temps, afin de rechercher l'existence d'une éventuelle diminution des taux parallèle à l'augmentation des concentrations sanguines, ainsi que l'ont décrit Pohland et Bernard (7). Quoi qu'il en soit, même en l'absence d'explication satisfaisante, il est clair que le paracétamol présente des phénomènes de redistribution alors même que sa lipophilie et son volume de distribution sont faibles. Pounder et Davies (22) mettent également en évidence une redistribution de la zopiclone, dont le $\mathrm{Vd}$ est de l'ordre de $1,5 \mathrm{~L} / \mathrm{Kg}$, au niveau du lobe hépatique gauche et du poumon gauche, redistribution très vraisemblablement en relation avec une diffusion depuis le tractus digestif. Un mécanisme similaire est évoqué dans deux études très récentes $(71,72)$ qui montrent que la mirtazapine, molécule basique à large volume de distribution, présente des concentrations en post-mortem équivalentes entre le sang cardiaque et le sang veineux fémoral, alors que les concentrations dans le parenchyme hépatique sont très nettement supérieures. Ces exemples démontrent clairement que la redistribution d'une molécule doit être interprétée en fonction d'un faisceau de paramètres, et non pas uniquement en fonction de sa lipophilie et/ou de son volume de distribution. Le mécanisme d'absorption doit naturellement être pris en compte dans l'interprétation des résultats, la diffusion depuis le tractus digestif étant susceptible de concerner n'importe quel type de molécule. D'autre part, certaines molécules peuvent, même si leur volume de distribution est faible, s'accumuler dans des organes pour lesquels elles présentent une affinité particulière (73), et être ensuite relarguées de ces organes en période post-mortem. Il s'agit naturellement des organes "réservoirs" tels que le foie ou le myocarde, mais aussi des muscles ou du tissu adipeux. Ainsi le paracétamol pourrait-il être progressivement relargué à partir du parenchyme hépatique dans le compartiment sanguin central sans que soit affecté le compartiment périphérique (70). Il est d'ailleurs intéressant de noter que le cerveau, organe pourtant très vascularisé, fait peu l'objet de phénomènes de redistribution. Les concentrations cérébrales de différentes molécules obtenues aussi bien chez l'homme que chez l'animal en période post-mortem ne sont que très modérément augmentées par rapport aux concentrations en sang veineux périphérique $(25,28,67)$. Par ailleurs, selon Moore et al. (74), les concentrations cérébrales sont homogènes pour des prélèvements effectués en différents sites corticaux, mais légèrement différentes pour des prélèvements effectués au niveau de la substance blanche, compte tenu vraisemblablement de différences liées à la teneur en eau de ces deux milieux. Ces résultats, obtenus avec une molécule très hydrosoluble, en l'oc- currence l'éthanol, devraient être confirmés avec d'autres molécules, en particulier les molécules lipophiles à large volume de distribution, pour pouvoir être pris en compte. Quoi qu'il en soit, il apparaît clairement que le cerveau est un milieu relativement protégé des phénomènes de redistribution. Ceci est très vraisemblablement dû au fait que la barrière hémato-encéphalique est, de par sa structure, beaucoup moins perméable que les autres membranes de l'organisme. En effet, les jonctions serrées entre les cellules endothéliales des capillaires cérébraux et la présence d'un manchon de cellules gliales autour de ces capillaires représentent un obstacle important au transfert des petites et moyennes molécules hydrosolubles. D'autre part, l'importante épaisseur cumulée des membranes à traverser et l'absence de protéines dans le liquide céphalo-rachidien et le liquide interstitiel ralentissent considérablement le transfert des grosses molécules liposolubles (73).

\section{> Métabolisme}

La poursuite du métabolisme de certaines molécules en période post-mortem est un point important à déterminer puisqu'il conditionne la dégradation de la molécule mère, mais aussi la synthèse de ses métabolites. Ce phénomène doit être distingué de la dégradation postmortem de certaines molécules sous l'influence des bactéries de la putréfaction. Nous disposons de peu de travaux expérimentaux ou d'observations dans ce domaine. Seul le métabolisme de la cocaïne en période post-mortem a fait l'objet de plusieurs études. Selon Hearn et al. (75), la diminution des concentrations de cocaïne en période post-mortem observée dans certains cas serait due à une poursuite du métabolisme. Compte tenu de l'acidification du $\mathrm{pH}$ plasmatique, l'hydrolyse en benzoylecgonine n'est vraisemblablement pas le phénomène prédominant, comme le confirment Moriya et Hashimoto (76). L'hypothèse d'une poursuite de l'action des estérases plasmatiques peut être alors envisagée. Selon Isenschmid et al. (77), la benzoylecgonine identifiée dans le sang post-mortem proviendrait d'une hydrolyse de la cocaïne à $\mathrm{pH}$ physiologique avant le décès, alors que l'ecgonine méthylester pourrait provenir d'une hydrolyse post-mortem sous l'action des estérases plasmatiques. McKinney et al. (78) confirment cette notion en montrant une élévation progressive des concentrations d'ecgonine méthylester en sang veineux fémoral après administration de chlorhydrate de cocaïne IV à un lot d'animaux. La poursuite de l'activité enzymatique des estérases plasmatiques en période post-mortem n'est toutefois pas clairement démontrée. D'autre part, selon Logan et al. (3), la cocainne pourrait être hydrolysée en benzoylecgonine en période post- 
mortem sous l'action de la cocaïne-méthylestérase hépatique. Les études concernant le cocaéthylène sont contradictoires. Selon Moriya et Hashimoto (76), une certaine proportion de cocaéthylène pourrait être synthétisée dans les heures qui suivent le décès chez l'animal, alors qu'il serait dégradé, plus lentement que la cocaïne, chez l'homme (79). Enfin, la superposition de phénomènes de redistribution entre différents sites de prélèvement, y compris au niveau de l'humeur vitrée (78), à la poursuite du métabolisme hépatique et plasmatique de la cocaïne rend l'interprétation de ces résultats particulièrement aléatoire (3). Ces résultats sont corroborés par une étude de Moriya et Hashimoto (80) montrant une poursuite du métabolisme d'un organophosphoré, le dichlorvos, sous l'action d'estérases plasmatiques et tissulaires. Ces études ont pour intérêt de montrer que la poursuite d'un métabolisme enzymatique est possible en période post-mortem précoce. De manière générale, le métabolisme des xénobiotiques est majoritairement hépatique et comprend deux types de réaction, les réactions dites de phase 1 et des réactions dites de phase 2. Les réactions de phase 1 dégradent la molécule mère ; il s'agit essentiellement de réactions d'oxydation ( $\mathrm{N}$-oxydations, $\mathrm{N}$-déméthylation, $\mathrm{O}$ déméthylation, etc..), de réduction ou d'hydrolyse. Ces réactions sont sous la dépendance de différentes enzymes dont les principales sont les mono-oxygénases liées au cytochrome P450. Ces mono-oxygénases sont constitués d'une hémoprotéine, le cytochrome $\mathrm{P} 450$, d'une enzyme réductrice, la NADPH-cytochrome-P450-réductase, d'une apoprotéine de composition variable, responsable de la diversité de ces mono-oxygénases, et de phosphatidylcholine (73). Les réactions de phase 2 sont des réactions de conjugaison visant à rendre plus hydrosoluble le métabolite formé afin de faciliter son élimination urinaire. Il s'agit de réactions de glucuroconjugaison sous l'action de l'UDP-glucuronyl transférase et de réactions de sulfoconjugaison, de méthylation, d'acétylation, de trans-sulfuration ou enfin de conjugaison au gluthation (73). Yamazaki et al. (81) ont étudié l'évolution l'activité de ces différents enzymes en période post-mortem chez le rat, montrant qu'elle diminuait en période post-mortem immédiate mais ne disparaissait totalement qu'au bout de plusieurs jours pour certaines enzymes, laissant donc persister des possibilités métaboliques durant cette période. Ils ont ainsi démontré que l'activité du cytochrome P450 diminuait de $92 \%$ en 48 heures, et que l'activité de la NADPH-cytochrome-P450-réductase diminuait dans les mêmes proportions. En revanche, l'activité de l'UDP-glucuronyl transférase diminue plus lentement, puisqu'il en subsistait environ $32 \%$ au bout de $48 \mathrm{~h}$. Ces résultats devraient être confirmés chez l'homme à partir d'études réalisées sur microsomes hépatiques prélevés à différents temps autopsiques. Ils mettent également l'accent sur la nécessité de doser dans la mesure du possible les métabolites de la molécule concernée, l'évolution du ratio molécule mère/métabolite pouvant être un élément d'orientation intéressant dans l'interprétation des résultats.

\section{> Élimination}

De même que pour l'absorption, aucune étude n'a été réalisée à notre connaissance sur l'évolution éventuelle dans les heures qui suivent le décès des mécanismes d'élimination. L'élimination urinaire comprend trois mécanismes : la filtration glomérulaire, la sécrétion et la réabsorption tubulaires. La filtration glomérulaire est directement conditionnée par le débit sanguin afférent au niveau des artères rénales. L'arrêt de toute circulation sanguine lors du décès devrait interrompre définitivement ce processus de filtration. La situation est plus complexe au niveau tubulaire. La sécrétion tubulaire est sous la dépendance d'un mécanisme actif nécessitant de l'ATP ; elle est donc vraisemblablement interrompue très rapidement après le décès. Par contre, la réabsorption tubulaire au niveau du tube contourné distal et du tube collecteur est sous la dépendance d'une diffusion passive. L'acidification du $\mathrm{pH}$ plasmatique et interstitiel peut donc modifier la réabsorption et entrấner un relargage d'acides faibles en particulier. La vésicule biliaire concentre et élimine essentiellement des grosses molécules lipophiles, ainsi que les dérivés glucurono- et mercaptoconjugués de certaines molécules. L'excrétion de ces molécules dans la bile primaire est sous la dépendance de différents mécanismes de transport actif qui sont très vraisemblablement interrompus lors de l'arrêt de la synthèse d'ATP. De même, le stockage de la bile primaire dans la vésicule biliaire et sa concentration par réabsorption active d'eau sont interrompus, comme est interrompue la vidange biliaire active dans le deuxième duodénum.

\section{Conséquences pratiques en toxicologie médico-légale}

D'un point de vue pratique, le respect de certaines précautions lors de la réalisation des prélèvements autopsiques est important pour limiter au maximum les erreurs d'interprétation. Il convient en particulier de disposer de multiples prélèvements, aussi bien de différents liquides biologiques que tissulaires, afin de pouvoir comparer les concentrations dans les différents secteurs. Les prélèvements sanguins doivent comporter des échantillons cardiaques et périphériques. Dans le 
cadre d'une étude de redistribution post-mortem, il convient d'individualiser les prélèvements provenant des cavités droites de ceux provenant des cavités gauches, afin, le cas échéant, de déterminer quel est le mécanisme de redistribution mis en cause. Du fait de l'importance des phénomènes de redistribution dont il fait l'objet, le sang cardiaque ne doit naturellement pas être utilisé pour estimer la dose absorbée. En ce qui concerne le sang périphérique, l'échantillon sanguin idéal est - selon Pounder (21) - réalisé immédiatement après le décès dans la veine fémorale, sur un vaisseau préalablement ligaturé afin d'éviter toute contamination par les veines iliaques et la veine cave inférieure. Les conditions habituelles de réalisation des autopsies rendent bien entendu un tel prélèvement extrêmement difficile à réaliser. D'autre part, même s'il est généralement plus représentatif que le sang cardiaque, le sang fémoral prélevé à l'autopsie présente souvent des concentrations plus élevées que celles retrouvées anteou péri-mortem (23). Enfin, et bien que cela soit extrêmement rare, il arrive que la concentration en sang veineux fémoral soit à peu près équivalente voire supérieure à celle mesurée dans le sang cardiaque. Ceci se produit généralement lorsque des manoeuvres de réanimation cardiaque ont été mises en œuvre avant le décès, provoquant vraisemblablement une chasse du sang cardiaque vers les gros vaisseaux $(23,24)$. Le même phénomène a été constaté par Hilberg et al. (82) qui, lors d'une expérimentation animale concernant la redistribution post-mortem de l'amitriptyline, ont euthanasié les animaux avec du chlorure de potassium, induisant une fibrillation ventriculaire susceptible de chasser le sang vers les gros vaisseaux. Il est également possible que le sang prélevé provienne en réalité de la veine cave inférieure à cause d'un "effet de siphon" lors du prélèvement (5). D'autre part, les seules sources de redistribution potentielle vers les vaisseaux fémoraux sont les tissus graisseux et les muscles (58). Enfin, il semblerait que les concentrations en sang veineux fémoral soient relativement stables dans le temps, contrairement aux autres sites (70). Le sang veineux sous-clavier ne doit pas être quant à lui retenu comme site périphérique pour les raisons précédemment évoquées. Ces résultats sanguins doivent naturellement être confrontés à ceux obtenus à partir des autres liquides biologiques, urine, liquide gastrique et bile. En ce qui concerne les prélèvements de viscères thoracoabdominaux (poumons, foie, reins), nous avons déjà évoqué le fait que, compte tenu de l'importance de la redistribution digestive, les concentrations étaient plus élevées au niveau du lobe hépatique gauche, du poumon gauche, voire du rein gauche qu'au niveau des autres lobes hépatiques ou du poumon et du rein droits.
Il semblerait - même si les différences ne sont pas statistiquement significatives - que l'apex pulmonaire gauche concentre moins les molécules basiques lipophiles que la base gauche. Il est donc préférable d'effectuer les prélèvements à ce niveau (83). Le cerveau ne semblant pas faire l'objet de phénomènes de redistribution particuliers, aucune recommandation précise quant à la localisation du prélèvement n'est donnée. D'autres milieux, réputés moins sensibles aux phénomènes de redistribution, ont également été proposés pour contourner ces difficultés d'interprétation. Il s'agit essentiellement de l'humeur vitrée, du muscle, de la moelle osseuse ou du liquide céphalo-rachidien. L'humeur vitrée est un milieu facile à collecter qui présente l'intérêt majeur d'être protégé des proliférations bactériennes et fongiques liées à la putréfaction, par une barrière anatomique, la sclérotique. L'humeur vitrée est ainsi considérée depuis longtemps comme un milieu de choix pour distinguer en période post-mortem l'éthanol d'origine exogène de l'éthanol formé par la fermentation bactérienne $(42,84,85)$. En ce qui concerne l'interprétation des concentrations d'éthanol retrouvées dans l'humeur vitrée, le rapport des concentrations humeur vitrée/sang variant de 0,9 à 1,38 , seule une approximation est envisageable $(42,86)$. L'humeur vitrée ne s'avère pas aussi discriminante pour d'autres molécules : McKinney et al. (78) ont mis en évidence une augmentation significative des concentrations de cocaïne ainsi qu'une tendance à l'augmentation de ceux de benzoylecgonine et d'ecgonine méthylester dans l'humeur vitrée dans les huit premières heures post-mortem. D'autre part, pour certaines molécules telles que la digoxine, aucune corrélation ne peut être établie entre les concentrations sanguines et les concentrations dans l'humeur vitrée (87). Le muscle a été également proposé comme milieu de dosage car il est généralement disponible en grandes quantités, qu'il est soumis plus tardivement que le sang aux phénomènes de putréfaction (88), et qu'il est d'autre part aisé d'obtenir des prélèvements à distance du compartiment central, donc des réservoirs gastrique et pulmonaire $(82,89)$. Le muscle est donc apparu assez rapidement comme un milieu possible pour les dosages quantitatifs, pour les amphétamines entre autres (2). Langford et al. (89) ont réalisé des dosages post-mortem de différentes molécules (témazépam, amitriptyline, paracétamol, propoxyphène) sur 12 sites musculaires différents. Les résultats montrent en fait une très grande variabilité entre les différents sites. Les concentrations les plus élevées sont retrouvées au niveau des sites musculaires proches du compartiment central, dans le diaphragme en particulier. D'autre part, cette variabilité entre les différents sites musculaires semble être plus 
importante pour les molécules basiques à large volume de distribution (88). Le délai entre la prise du toxique et le décès serait également un facteur à prendre en compte dans l'interprétation des résultats. En effet, si ce délai est rapide, la molécule est encore dans la période d'absorption et la concentration dans le muscle peut être encore peu élevée, et inversement si ce délai est trop long $(88,90)$. Le muscle est donc vraisemblablement le siège de phénomènes de redistribution d'intensité variable. Le prélèvement musculaire doit donc être réalisé en périphérie (grand dentelé, long supinateur) et limité à la recherche et à l'identification des xénobiotiques. La moelle osseuse a fait l'objet de quelques travaux. Très vascularisée et riche en lipides, la moelle osseuse concentre un grand nombre de molécules. Elle est d'autre part très protégée des contaminations bactériennes de par ses rapports anatomiques $(91,92)$. Elle a été longtemps considérée utilisable uniquement à titre qualitatif en l'absence d'autres milieux de prélèvements, jusqu'à ce que Winek et al. (93) démontrent qu'il existe chez le lapin une bonne corrélation entre les taux sanguins et médullaires de nortriptyline, mais uniquement pendant les 24 premières heures suivant le décès, les concentrations médullaires augmentant régulièrement au delà de ce délai. Au contraire; Hilberg et al. (82) ne retrouvent aucune corrélation entre les concentrations sanguines et médullaires (sternum et fémur) d'amitriptyline chez le cochon. De plus, les concentrations retrouvées lors des prélèvements médullaires effectués au niveau sternal avant et après l'euthanasie étaient notablement différents, et n'étaient pas non plus corrélés à ceux retrouvés lors des prélèvement fémoraux, indiquant donc un phénomène de redistribution. Il n'est donc pas possible de recommander la moelle comme milieu alternatif. Le LCR a également été proposé comme milieu alternatif. Mais un grand nombre de toxiques ne diffusent pas dans le LCR. Très peu d'études ont été menées à ce jour pour apprécier l'évolution des concentrations des xénobiotiques dans ce milieu, et déterminer éventuellement le rapport des concentrations des molécules concernées avec leur concentration sanguine. Selon Logan et Smirnov (26), qui ont réalisé des dosages de morphine dans le LCR, le sang périphérique et ventriculaire, sur une série de 32 décès impliquant cette molécule, si le taux de morphine semble stable chez un même cadavre dans les heures qui suivent l'autopsie, le rapport des concentrations LCR/sang cardiaque ou périphérique est trop variable d'un cadavre à l'autre pour être utilisable. Selon Hilberg et al. (82), l'amitriptyline présenterait une redistribution vers le LCR dans les heures qui suivent le décès. Enfin, le liquide hématique issu des gouttières pleurales est certainement le plus mauvais milieu biologique car il s'agit d'un mélange de sang et de liquides séreux issus des poumons et autres organes thoraciques, voire même de l'estomac, qui ont pu diffuser après le décès. Nous n'aborderons pas ici le problème du traitement de l'échantillon qui sort du cadre traité, mais il est évident que le non respect des conditions habituelles de conservation (adjonction de $\mathrm{NaF}$ à une partie des échantillons sanguins, conservation au froid) crée une source d'erreurs supplémentaire.

\section{Conclusion et perspectives}

Les phénomènes de redistribution rendent parfois difficile l'interprétation des résultats des analyses toxicologiques réalisées sur des échantillons recueillis en période post-mortem, d'autant plus volontiers que plusieurs mécanismes différents peuvent entrer en compétition $(3,94)$. En pratique, le problème le plus fréquemment rencontré est celui d'une disparité des concentrations d'une même molécule entre différents sites de prélèvement. Si ces différences sont modérées, et que toutes les concentrations restent dans une fenêtre soit d'ordre thérapeutique (ou non toxique pour les molécules non médicamenteuse), soit d'ordre toxique, l'interprétation ne soulèvera pas de difficultés majeures. Les difficultés d'interprétation surviennent en revanche lorsque les concentrations sont franchement discordantes entre les différents sites et/ou à différents temps de prélèvement. L'interprétation des résultats doit alors tenir compte des caractéristiques physico-chimiques de la molécule, ainsi que, dans la mesure du possible, des données de l'autopsie, parfois essentielles pour expliquer certaines différences. Ainsi la position du corps, ou la régurgitation éventuelle du contenu gastrique dans les voies aériennes supérieures peuvent être des éléments d'orientation intéressants, de même que, naturellement, l'état de décomposition éventuelle du corps. La connaissance de ces phénomènes de redistribution relativise la fiabilité des banques de données de concentrations toxiques et létales réalisées à partir de prélèvements autopsiques réalisés dans des conditions inconnues, particulièrement en ce qui concerne les sites de prélèvement et le délai écoulé entre le décès et les prélèvements. Ceci est d'autant plus important qu'un grand nombre de molécules rencontrées en toxicologie médico-légale sont des bases faibles lipophiles à large volume de distribution, donc fortement suspectes de redistribution. D'autre part, on ne dispose que de très peu d'informations quant à l'influence des autres paramètres pharmacocinétiques des xénobiotiques sur les phénomènes de redistribution, ainsi que sur l'évolution des capacités métaboliques de l'organisme dans les premières heures après le décès. Le champ d'investiga- 
tions futur est donc large. Il serait nécessaire de disposer d'études visant à déterminer réellement si certaines capacités d'absorption, de métabolisation ou d'élimination persistent en période post-mortem immédiate, et d'autre part de constituer des banques de données concernant l'étendue de la redistribution post-mortem des différentes molécules en fonction de leurs paramètres pharmacocinétiques, ceci bien entendu à partir d'études expérimentales dont le modèle reste à déterminer. Nous pensons enfin qu'il est nécessaire de quantifier - probablement sous forme de pourcentage - l'intensité des phénomènes de redistribution. La littérature montre que certains auteurs évoquent un phénomène de redistribution pour des variations minimes entre les différents sites et/ou entre les différents temps de prélèvement, alors que d'autres n'évoquent ce phénomène que devant des variations beaucoup plus importantes. Des variations minimes n'engendrent d'ailleurs pas, ainsi que cela a déjà été évoqué, de grandes difficultés d'interprétation. La difficulté est de déterminer premièrement quel site et quel temps de prélèvement doivent être considérés comme références, et deuxièmement à partir de quel pourcentage de variation devra-t-on considérer qu'il y a effectivement redistribution. Le débat reste ouvert...

\section{Références}

1. Skopp G., Lutz R., Ganßmann B., Mattern R., Aderjan R. Postmortem distribution pattern of morphine and morphine glucuronides in heroin overdose. Int. J. Legal Med. $1996 ; 109: 118-24$.

2. Nagata T., Kimura K., Hara K., Kudo K. Methamphetamine and amphetamine concentrations in postmortem rabbit tissues. Forensic Sci. Int. 1990 ; 48 : 39-47.

3. Logan B.K., Smirnow D., Gullberg R.G. Lack of predictable site-dependent differences and time-dependent changes in postmortem concentrations of cocaine, benzoylecgonine and cocaethylene in humans. J. Anal. Toxicol. $1997 ; 20: 23-31$.

4. Hilberg T., Bugge A., Beylich K.M., Ingum J., Bjorneboe A., Morland $\mathbf{J}$. An animal model of postmortem amitriptyline redistribution. J. Forensic Sci. 1993 ; 38 : 81-90.

5. Pounder D.J.; Smith D.R.W. Postmortem diffusion of alcohol from the stomach. Am. J. Forensic Med. Pathol. $1995 ; 16: 89-96$.

6. Fuke C., Berry C.L., Pounder D.J. Postmortem diffusion of ingested and aspirated paint thinner. Forensic Sci. Int. 1996 ; 78 : 199-207.

7. Pohland R., Bernhard N.R. Postmortem serum and tissue redistribution of fluoxetine and norfluoxetine in dogs following oral administration of fluoxetine hydrochloride (Prozac®). J. Forensic Sci. 1997 ; 42 : 812-6.
8. Pounder D.J., Fuke C., Cox D.E., Smith D., Kuroda N. Postmortem diffusion of drugs from gastric residue. Am. J. Forensic. Med. Pathol. $1996 ; 17: 1-7$.

9. Marraccini J.V., Carroll T., Grant S., Halleran S., Benz J.A. Differences between multisite postmortem ethanol concentrations as related to agonal events. J. Forensic Sci. 1990 ; 35 : 1360-6.

10. Pounder D.J. Postmortem diffusion of tracheal lidocaine into heart blood following intubation for cardiopulmonary resuscitation. J. Forensic Sci. 1997 ; 42 : 965-6.

11. Pounder D.J., Yonemitsu K. Postmortem absorption of drugs and ethanol from aspirated vomitus - an experimental model. Forensic Sci. Int. 1991 ; 51 : 189-95.

12. MacIntyre A.C., Cutler D.J. The potential role of lysosomes in tissue distribution of weak bases. Biopharm. Drug. Dispos. 1988 ; 9 : 513-26.

13. Miyazaki T., Kojima T., Yashiki M., Wakamoto H., Iwasaki Y., Taniguchi T. Site dependence of methamphetamine concentrations in blood samples collected from cadavers of people who had been methamphetamine abusers. Am. J. Forensic Med. Pathol. 1993 ; 14 : 121-4.

14. Daniel W.A., Bickel M.H., Honegger U.E. The contribution of lysosomal trapping in the uptake of desipramine and chloroquine by different tissues. Pharmacol. Toxicol. $1995 ; 77: 402-6$.

15. Pla A., Hernandez A.F., Gil F., Garcia-Alonso M., Villanueva $E$. A fatal case of oral ingestion of methanol. Distribution in postmortem tissues and fluids including pericardial fluid and vitreous humor. Forensic Sci. Int. 1991 ; 49 : 193-6.

16. Hilberg T., Morland J., Bjorneboe A. Postmortem release of amitriptyline from the lungs; a mechanism of postmortem drug redistribution. Forensic Sci. Int. 1994 ; 64 : 47-55.

17. Moriya F., Hashimoto Y. Postmortem diffusion of tracheal lidocaine into heart blood following intubation for cardiopulmonary resuscitation. J. Forensic Sci. 1997 ; $42: 296-9$.

18. Pounder D.J., Owen V., Quigley C. Postmortem changes in blood amitriptyline concentration. Am. J. Forensic Med. Pathol. 1994 ; 15 : 224-30.

19. Moriya F., Hashimoto Y. Redistribution of basic drugs into cardiac blood from surrounding tissues during early stages postmortem. J. Forensic Sci. 1999 ; 44 : 10-6.

20. Hilberg T., Bugge A., Beylich K.M., Morland J., Bjorneboe A. Diffusion as a mechanism of postmortem drug redistribution : an experimental study in rats. Int. J. Legal Med. 1992 ; 105 : 87-91.

21. Pounder D.J. The nightmare of postmortem drug changes. In : Wecht C.H., ed. Legal medicine 1993. New Hampshire : Butterworth Legal Publishers, 1994 ; 16393.

22. Pounder D.J., Davies J.I. Zopiclone poisoning: tissue distribution and potential for postmortem diffusion. Forensic Sci. Int. $1994 ; 65$ : 177-83.

23. Hilberg T., Rogde S., Morland J. Postmortem drug redistribution - human cases related to results in experimental animals. J. Forensic Sci. 1999 ; 44 : 3-9. 
24. Prouty R.W., Anderson W.H. The forensic science implications of site and temporal influences on postmortem blood-drug concentrations. J. Forensic Sci. 1990 ; 35 : 243-70.

25. Sawyer W.R., Forney R.B. Postmortem disposition of morphine in rats. Forensic Sci. Int. 1988 ; 38 : 259-73.

26. Logan B.K., Smirnow D. Postmortem diffusion and redistribution of morphine in man. J. Forensic Sci. $1996 ; 41: 37-46$.

27. Barnhart F.E., Fogacci J.R., Reed D.W. Methamphetamine - a study of postmortem redistribution. J. Anal. Toxicol. $1999 ; 23: 69-70$.

28. Jones G.R., Pounder D.J. Site dependence of drug concentrations in postmortem blood - a case study. $\mathrm{J}$. Anal. Toxicol. $1987 ; 11: 186-90$.

29. Cotran R.S., Kumar V., Robbins S.L., eds. Cellular Injury and adaptation. In : Robbin's pathologic basis of disease. 4th ed. Piladelphia : WB Saunders, 1989 ; 1-38.

30. Langford A.M., Pounder D.J. Possible markers for postmortem drug redistribution. J. Forensic Sci. 1997 ; 42 : 88-92.

31. Skopp G., Lutz R., Pötsch L., Ganßmann B., Klinder K., Schmidt A., Aderjan R., Mattern R. An in vitro experiment for postmortem vascular permeation. The passage of morphine and morphine glucuronides accross a vascular wall. J. Forensic Sci. 1997 ; 42 : 486-91.

32. Thomsen H., Kaatsch H.J., Krisch B. How and why does the platelet count in postmortem blood change the early postmortem interval ? J. Forensic Sci. 1999 ; 101 : 185-94.

33. Durigon M. Pratique médico-légale. Paris : Masson, $1999: 39-49$.

34. Fallani M. Contributo allo studio della circolazione ematica post-mortale. Minerva Medicoleg. 1961 ; 81 : 108-15.

35. Gomez-Zapata M., Alcaraz M., Luna A. Studies on postmortem circulation of the blood. Z. Rechtsmed. 1989 ; $103: 27-32$.

36. O'Neal C.L., Poklis A. Postmortem production of ethanol and factors that influence interpretation : a critical review. Am. J. Forensic Med. Pathol. 1996 ; 17 : 8-20.

37. Corry J.E.L. Possible sources of ethanol ante- and postmortem: its relationship to the biochemistry and microbiology of decomposition. J. Appl. Bacteriol. 1978 ; 44 : $1-56$.

38. Bouillerot A., Laviano-Rousselin C. Dosage d'éthanol : les erreurs pré-analytiques. Alcools et Glycols. Journée thématique de la Société Française de Toxicologie Analytique. Paris, 8 décembre 1999.

39. Kupfer D.M., Chaturvedi A.K., Canfield D.V., Roe B.A. PCR-based identification of postmortem microbial contaminants - a preliminary study. J. Forensic Sci. $1999 ; 44$ : 592-6.

40. Alexander W.D. Postmortem urinary alcohol is unreliable in diabetes. B.M.J. $1998 ; 317: 206$.

41. Briglia E.J., Bidanset J.H., Dal Cortivo L.A. The distribution of ethanol in postmortem blood specimens. J. Forensic Sci. 1992 ; 37 : 991-8.

42. Deveaux M. Alcool éthylique. In : Kintz P., ed. Toxicologie et pharmacologie médicolégales. Paris : Elsevier, 1998 ; 111-26.
43. Gilliland M.G., Bost R.O. Alcohol in decomposed bodies : postmortem synthesis and distribution. $J$. Forensic Sci. 1993 ; 38 : 1266-74.

44. Grellner W., Iffland R. Assessment of postmortem blood alcohol concentrations by ethanol levels measured in fluids from putrefactive blisters. Forensic Sci. Int. 1997 ; $90: 57-63$.

45. Jones A.W., Andersson R., Sakshaug J., Morland J. Possible formation of ethanol in postmortem blood specimen after antemortem treatment with mannitol. J. Anal. Toxicol. $1991 ; 15: 157-8$.

46. Laviano C. Production et consommation d'éthanol postmortem dans deux liquides biologiques. Ann. Biol. Clin. 1998 ; $56: 96-9$.

47. Takayasu T., Ohshima T., Tanaka N., Maeda H., Kondo T., Nishigami J., Nagano T. Postmortem degradation of administered ethanol-d6 and production of endogenous ethanol: experimental studies using rats and rabbits. Forensic Sci. Int. $1995 ; 76: 129-40$.

48. Robertson M.D., Drummer O.H. Postmortem distribution and redistribution of nitrobenzodiazepines in man. $\mathbf{J}$. Forensic Sci. 1998 ; 43 : 9-13.

49. Ballantyne B., Bright J.E., Williams P. The post-mortem rate of transformation of cyanide. Forensic Sci. 1974 ; $3: 71-6$.

50. Chiou W.L. The phenomenon and rationale of marked dependence of drug concentration on blood sampling site. Implications in pharmacokinetics, pharmacodynamics, toxicology and therapeutics (Part I). Clin. Pharmacokinet. $1989 ; 17: 175-99$.

51. Elsirafy A.A., Ghanem A.A., Eid A.E., Eldakroory S.A. Chronological study of diazinon in putrefied viscera of rats using GC/MS, GC/EC, and TLC. Forensic Sci. Int. $2000 ; 109$ : 147-57.

52. Tillement J.P., Lindenlaub E. Protein binding and drug transport. Stuttgart : FK Schattauer Verlag, 1986.

53. Konikova A.S., Vinarskaya A.A., Nikulin V.I., Pogossova A.V., Petukhova L.M. Protein degradation to low-molecular compouds after death and during reanimation. Virchows Arch. B. Cell. Pathol. $1975 ; 18$ : 347-55.

54. Bonte W., Bleifuss J., Volck J. Experimental investigations in post-mortem protein degradation. Forensic Sci. $1976 ; 7: 9-22$.

55. Oehmichen M., Gencic M. Postmortal diffusion of plasma albumin in rat brain. Z. Rechtsmed. $1980 ; 84$ : 113-23.

56. Tomson T., Sköld A.C., Holmgren P., Nilsson L., Danielsson B. Postmortem changes in blood concentrations of phenytoin and carbamazepine: an experimental study. Ther. Drug Monit. 1998 ; 20 : 309-12.

57. Skopp G., Potsch L., Ganssmann B., Aderjan R., Mattern $R$. A preliminary study on the distribution of morphine and its glucuronides in the subcompartiments of blood. J. Anal. Toxicol. $1998 ; 22: 261-4$.

58. Cook D.S., Braithwaite R.A., Hale K.A. Estimating antemortem drug concentrations from postmortem blood samples: the influence of postmortem redistribution. $\mathrm{J}$. Clin. Pathol. $2000 ; 53$ : 282-5. 
59. Yonemitsu K., Pounder D.J. Postmortem toxico-kinetics of co-proxamol. Int. J. Legal Med. 1992 ; 104 : 347-53.

60. Martin A., Pounder D.J. Post-mortem toxico-kinetics of trazodone. Forensic Sci. Int. $1992 ; 56: 201-7$.

61. Pounder D.J., Hartley A.K., Watmough P.J. Postmortem redistribution and degradation of dothiepin. Am. J. Forensic Med. Pathol. 1994 ; 15 : 231-5.

62. Keller T., Schneider A., Tutsch-Bauer E. Fatal intoxication due to dothiepin. Forensic Sci. Int. $2000 ; 109: 159-66$.

63. Jaffe P.D., Batziris H.P., van der Hoeven P., DeSilva D., McIntyre I.M. A study involving venlafaxine overdoses : comparison of fatal and therapeutic concentrations in postmortem specimens. J. Forensic Sci. 1999 ; 44 : 193-6.

64. Levine B., Wu S.C., Dixon A., Smialek J.E. Site dependence of postmortem blood methadone concentrations. Am. J. Forensic Med. Pathol. 1995 ; 16 : 97-100.

65. Milroy C.M., Forrest A.R. Methadone deaths: a toxicological analysis. J. Clin. Pathol. $2000 ; 53: 277-81$.

66. O'Sullivan J.J., McCarthy P.T., Wren C. Differences in amiodarone, digoxin, flecainide and sotalol concentrations between antemortem serum and femoral postmortem blood. Human Exp. Toxicol. 1995 ; 14 : 605-8.

67. Hilberg T., Ripel A., Slordal L., Bjorneboe A., Morland J. The extent of postmortem drug redistribution in a rat model. J. Forensic Sci. 1999 ; 44 : 956-62.

68. Gerostamoulos J., Drummer O.H. Postmortem redistribution of morphine and its metabolites. J. Forensic Sci. $2000 ; 45: 843-5$.

69. Carrupt P., Testa B., Bechalany A., El Tayar N., Descas P., Perisoud D. Morphine-6-glucuronide and morphine3 -glucuronide as molecular chameleons with unexpected lipophilicity. J. Med. Chem. 1991 ; 34 : 1272-5.

70. Gomez H.F., McKinney P., Phillips S., Roberts D.V., Brent J., Watson W.A. Postmortem acetaminophen pharmacokinetics: an experimental study of site and timedependent concentration changes. J. Forensic Sci. 1995; $40: 980-2$.

71. Anderson D.T., Fritz K.L., Muto J.J. Distribution of mirtazapine (Remeron ${ }^{\circledR}$ ) in thirteen postmortem cases. J. Anal. Toxicol. $1999 ; 23: 544-8$.

72. Moore K.A., Levine B., Smith M.L., Saki S., Schames J., Smialek J.E. Tissue distribution of mirtazapine (Remeron $\left.{ }^{\circledR}\right)$ in postmortem cases. J. Anal. Toxicol. 1999 ; $23: 541-3$.

73. Marquet P., Lachâtre G. Devenir des xénobiotiques dans l'organisme. In : Kintz P., ed. Toxicologie et pharmacologie médicolégales. Paris : Elsevier, 1998 ; 27-66.

74. Moore K.A., Kunsman G.W., Levine B.S., Herman M.M., Cervenak J., Hyde T.M. A comparison of ethanol concentrations in the occipital lobe and cerebellum. Forensic Sci. Int. 1997 ; 86 : 127-34.

75. Hearn W.L., Keran E.E., Wei H., Hime G. Site-dependent postmortem changes in blood cocaine concentrations. J. Forensic Sci. 1991 ; 36 : 673-84.

76. Moriya F., Hashimoto Y. The effect of postmortem interval on the concentrations of cocaine and cocaethylene in blood and tissues: an experiment using rats. J. Forensic Sci. 1996 ; 41 : 129-33.
77. Isenschmid D.S., Levine B.S., Caplan Y.H. The role of ecgonine methyl ester in the interpretation of cocaine concentrations in postmortem blood. J. Anal. Toxicol. $1992 ; 16: 319-24$.

78. McKinney P.E., Phillips S., Gomez H.F., Brent J., MacIntyre M., Watson W.A. Vitreous humor cocaine and metabolite concentrations: do postmortem specimens reflect blood levels at the time of death? J. Forensic Sci. $1995 ; 40: 102-7$.

79. Moriya F., Hashimoto Y. Postmortem stability of cocaine and cocaethylene in blood and tissues of humans and rabbits. J. Forensic Sci. 1996 ; 41 : 612-6.

80. Moriya F., Hashimoto Y. Comparative studies on tissue distributions of organophosphorus, carbamate and organochlorine pesticides in decedents intoxicated with these chemicals. J. Forensic Sci. 1999 ; 44 : 1131-5.

81. Yamazaki M., Wakasugi C. Postmortem changes in drugmetabolizing enzymes of rat liver microsome. Forensic Sci. Int. $1994 ; 67: 155-68$.

82. Hilberg T., Ripel A., Smith A.J., Slordal L., Morland J., Bjorneboe A. Postmortem amitriptyline pharmacokinetics in pigs after oral and intravenous routes of administration. J. Forensic Sci. 1998 ; 380-7.

83. Pounder D.J., Adams E., Fuke C., Langford A. Site to site variability of postmortem drug concentrations in liver and lung. J. Forensic Sci. 1996 ; 41 : 927-32.

84. Deveaux M., Gosset D. Le vitré: un milieu inconnu en toxicologie médico-légale. Toxicorama. $1996 ; 8$ : 15-20.

85. De Lima I.V., Midio A.F. Origin of blood ethanol in decomposed bodies. Forensic Sci. Int. 1999 ; 106 : 15762.

86. Pounder D.J., Kuroda N. Vitreous alcohol is of limited value in predicting blood alcohol. Forensic Sci. Int. $1994 ; 65: 73-80$.

87. Vorpahl T.E., Coe J.I. Correlation of antemortem and postmortem digoxin levels. J. Forensic Sci. 1978 ; 23 : 329-34.

88. Williams K.R., Pounder D.J. Site-to-site variability of drug concentrations in skeletal muscle. Am. J. Forensic Med. Pathol. 1997 ; 18 : 246-50.

89. Langford A.M., Taylor K.K., Pounder D.J. Drug concentration in selected skeletal muscles. J. Forensic Sci. 1998 ; $43: 22-7$.

90. Garriott J.C. Skeletal muscle as an alternative specimen for alcohol and drug analysis. J. Forensic Sci. 1991 ; 36 : 60-9.

91. Noguchi T.T. Drug analysis in skeletonizing remains. J. Forensic Sci. 1978 ; 23 : 490-2.

92. Winek C.L., Westwood S.E., Wahba W.W. Plasma versus bone marrow desipramine : a comparative study. Forensic Sci. Int. $1990 ; 48: 49-57$.

93. Winek C.L., Morris E.M., Wahba W.W. The use of bone marrow in the study of postmortem redistribution of nortriptyline. J. Anal. Toxicol. $1993 ; 17$ : 93-8.

94. Yonemitsu K., Pounder D.J. Postmortem changes in blood tranylcypromine concentration: competiting redistribution and degradation effects. Forensic Sci. Int. $1993 ; 59: 177-84$. 